\section{The efficacy and safety of fractional erbium yag laser combined with topical amniotic membrane stem cell (AMSC) metabolite product for facial rejuvenation: A controlled, split-face study}

\author{
Ni Putu Susari Widianingsih ${ }^{1}$ \\ Trisniartami Setyaningrum, ${ }^{2}$ \\ Cita Rosita S. Prakoeswa ${ }^{2}$ \\ ${ }^{1}$ Surabaya Skin Centre Clinic; \\ ${ }^{2}$ Dermatology and Venereology \\ Department of Dr Soetomo Hospital, \\ Surabaya, Indonesia
}

\begin{abstract}
Skin aging is a complex biological process, and it growing fast in number. Fractionated laser has been considered as the gold standard for skin rejuvenation. Stem cell-based therapy also becoming a promising new approach for treating this condition. To investigate the effect of fractional erbium laser and topical amniotic membrane stem cell (AMSC)-metabolite product (MP) on skin aging. A total of 9 female participants, were included in controlled split-face study. The participant's face was randomly allocated to AMSC-MP or saline, immediately after fractional laser procedure. All participants received 3 sessions of treatment with 4-weeks intervals. An evaluation was done using pre- and post- treatment photographs and skin analyzer, Janus. Laser-assisted transdermal delivery drug using topical AMSC-MP give a better improvement on skin pore and spot UV compared with the control group. The wrinkle and skin tone showed different results maybe because of multifactorial factors influence. No serious adverse events were found. This study suggest that fractional erbium YAG laser treatment combined with topical AMSC-MP is effective and safe for facial rejuvenation in Asian patients.
\end{abstract}

\section{Introduction}

Skin aging is a common and complex biological process, characterized by wrinkles, epidermal and dermal atrophy, rough texture, pigmentation, telangiectasia, and skin laxity. It mainly results from chronological aging caused by factors in the internal environment such as heredity and pho- toaging induced by factors in the external environment such as ultraviolet radiation. Therefore, increasing attention have focused on the treatment of facial rejuvenation, and it is necessary to explore more effective treatment. ${ }^{1}$

Stem cell-based therapy is becoming a promising new approach in almost every medical specialist. The use of growth factors in skin rejuvenation is emerging as a novel anti-aging treatment. ${ }^{2}$ However, stem cells-based therapies face several challenges including rejection and tumor transformation. As an alternative, the placenta offers an extraordinary source of fetal stem cells, including the amniotic epithelial cells (AECs), which retain some of the characteristics of embryonic stem cells, but show low immunogenicity, together with immunomodulatory and anti-inflammatory activities. ${ }^{3}$ Amniotic epithelial cells or amniotic membrane stem cells (AMSC) can be collected from different fetal annexes (amnion, chorion, amniotic fluid, Wharton jelly) and have to be safe, easy to collect, and devoid of immunogenic and tumorigenic properties. AMSC are collected from epithelial layer of the amnion which derives directly from the epiblast as it retains some ESC (embryonic stem cells) characteristic. ${ }^{3}$

Hydrophilic molecules larger than 500 Da have poor penetration through the stratum corneum. Most growth factor are large hydrophilic molecules greater than $20 \mathrm{kDA}$; therefore, they are unlikely to penetrate the epidermis in measurable quantities to produce pharmacologic effects. ${ }^{2}$

Transepidermal drug delivery (TED) is a new potential method in dermatology. Permeability alterations produced by ablative fractional resurfacing have been described with the aim to increasing the delivery of different substances into the skin. ${ }^{4}$ Fractionated lasers have acceptable aesthetic outcomes with reduced side effects for the patient. This technology could be applied for other uses such as laser assisted transdermal delivery (TDD). This technology has been demonstrated as feasible in an in vitro setting. ${ }^{5}$ The purpose of the fractionated laser is to perforate the stratum corneum; however this may mean damage to the underlying epidermis. Epidermal disruption is the main contributor to many of the side effects that are found with fractional lasers, ranging from erythema, oedema, blistering, and rarely infections. In addition, some patients have developed pigmentary changes, and thus caution is advised when attempting to treat darker Fitzpatrick IV to VI skins. While many of the adverse effects are reversible, pigmentary changes can sometimes be permanent.

A fractional $\mathrm{CO}_{2}$ laser treatment fascili-
Correspondence: Ni Putu Susari Widianingsih, Surabaya Skin Centre Clinic, Jl. Prof. Dr. Moestopo 175 Surabaya, Indonesia. Tel: +6231-5999595.

E-mail: susy_vq@yahoo.com, sbyskincentre@yahoo.co.id

Key words: Skin photoaging, AMSC-MP, laser-assisted transdermal delivery, fractional erbium Yag laser, skin rejuvenation.

Contributions: The Authors contributed equally.

Conflict of interest: Dr. Widianingsih has nothing to disclose.

Received for publication: 1 February 2119. Accepted for publication: 11 February 2119.

This work is licensed under a Creative Commons Attribution-NonCommercial 4.0 International License (CC BY-NC 4.0).

(C) Copyright N.P.S. Widianingsih et al., 2019 Licensee PAGEPress, Italy

Dermatology Reports 2019; 11(s1):8036

doi:10.4081/dr.2019.8036

tates the deep delivery of topical medication into the skin. However, ablative fractional erbium-doped yttrium aluminum garnet (Er-YAG) laser-assisted drug treatment may be superior to $\mathrm{CO}_{2}$ fractional laser treatment because it may have fewer side effects (such as post-inflammatory hyperpigmentation), while they have a common therapeutic concept of ablative fractional resurfacing for drug delivery. ${ }^{6}$

A combined use of fractional laser and other skin damage treatments such as microneedling therapy, radiofrequency waves were also proposed in order to achieve the best efficacy of treatment ${ }^{2,7}$, however, whether the application of topical AMSC metabolite product and the use of fractional erbium YAG laser can be combined in clinical use for facial rejuvenation had not been realized until recently.

\section{Objective}

The purpose of this study was to investigate the efficacy and safety of fractional erbium Yag laser combined with topical Amniotic Membrane Stem Cell Metabolite Product (AMSC-MP) for facial rejuvenation.

\section{Materials and Methods}

A total of 9 female participants, were included in controlled split face study, in Surabaya Skin Centre Clinic during the period from November 2016 to April 2017. 
All were healthy nonsmokers showing clinical signs of photoaging. Ages eligible for this study range from 40 to 60 years of age. One side of each subject's cheek was treated with fractional erbium YAG laser plus saline solution, and the other side was treated with fractional erbium YAG laser (action II fractional erbium laser by Lutronic) plus topical application of AMSC (amniotic membrane stem cell) or saline. The laser settings were $11 \mathrm{~J} / \mathrm{cm} 2$ in power, 2 multi shots, $0.5 \mathrm{sec}$ interval, at one single pass. All participants received 3 session of treatment were repeated at 4 -week intervals. An evaluation were done at baseline, four, eight and twelve weeks after the last treatment, using pre- and post- treatment photographs and skin analyzer, Janus.

\section{Results}

All 9 participants completed the 24week study protocol. Four participants were in II category of Glogau's scale skin aging, and the 5 participants were in III category. All participants were woman $(100 \%)$, age range 41 to 57 years old (mean age 49.78 \pm 6.01$)$.

Twelve weeks after the $3^{\text {rd }}$ treatment, both fractional erbium YAG laser plus AMSC and fractional erbium YAG laser plus saline solution showed improvement of pores, wrinkles, spot UV and skin tone.

\section{Pore improvement}

Baseline of pore grade from skin analyzer, Janus, showed no significant different between control side with mean values $48.33 \pm 4.21$ and intervention side with mean $50.00 \pm 5.59$ (table no.1 and no.2). Decreasing of the pore grade showed from

Table 1. Baseline characteristics of sample in Surabaya Skin Centre Clinic

\begin{tabular}{lc} 
Variable & $\begin{array}{c}\text { Normal Saline } \\
\text { and AMSC-PM ( } \mathrm{n}=9)\end{array}$ \\
Sex & \\
Male, n (\%) & $9(0)$ \\
Female, $\mathrm{n}(\%)$ & $9(100)$ \\
Glogau & \\
Category II & $4(44.44)$ \\
Category III & $5(55.55)$ \\
\hline Occupation & \\
Teacher, n (\%) & $2(22.22)$ \\
Housewife, $\mathrm{n}(\%)$ & $3(33.33)$ \\
Entrepreneur, $\mathrm{n}(\%)$ & $1(11.11)$ \\
Nurse, $\mathrm{n}(\%)$ & $2(22.22)$ \\
Employee, $\mathrm{n}(\%)$ & $1(11.11)$ \\
\hline
\end{tabular}

Notes: PM-AMSC: amniotic membrane stem cell-product metabolite, SD: standart deviation. mean values $\quad 50.00 \pm 5.59$ became $47.22 \pm 3.46$ at intervention side, and from $48.33 \pm 3.69$ became $47.11 \pm 3.69$ at control side. The average of dilated pore grade decreasing at AMSC side is greater than control side, with mean 2.78 for treatment side and 1.22 for control side. The mean values for pores were 50,0 before treatment and 47,0 after treatment for fractional erbium YAG laser with AMSC side. The mean values for pores on the non AMSC side were 48.3 before treatment and 47.2 after treatment. The pores on intervention sides was decreased after 24 weeks of observation. Comparing to the control sides was also decreased but it was greater on AMSC sides.

\section{Wrinkle improvement}

The wrinkle grade before treatment was different between the control side and intervention, with the mean grade at $19.78 \pm 3.90$ (control) and $25.11 \pm 14.84$ (intervention). Overall, the mean value of wrinkle grade at control side was decrease after treatment, with the mean $19.78 \pm 3.90$ (before treatment) became $18.67 \pm 4.64$ (after treatment). However, there was a very slight increased of the wrinkle grade at the intervention side with the mean value at $25.11 \pm 14.84$ (before treatment) and $25.56 \pm 25.16$ (after treatment).

\section{Pigmentation improvement}

Baseline data of the spot (UV) grade using skin analyzer, Janus (see Table 2), showed almost the same value before treat- ment between control side (mean at $13.11 \pm 6.99)$ and intervention side (mean at $14.22 \pm 8.06$ ). The spot (UV) grade on control side decrease at average mean $13.11 \pm 6.99$ before treatment and $11.44 \pm 4.07$ after treatment. The spot (UV) grade of intervention side shows decrease at average mean $14.22 \pm 8.06$ (before treatment) became $10.44 \pm 3.17$ (after treatment).

\section{Skin tone improvement}

The skin tone grade at baseline from skin analyzer, Janus, showed no difference between control side and intervention side, with mean values at $44.11 \pm 2.71$ and $44.78 \pm 7.45$ (before treatment). The skin tone grade at control side was increasing from the mean value at $44.11 \pm 2.71$ (before) became $46.22 \pm 1.79$ (after treatment). However, there was decreasing at intervention side from the mean value at $44.78 \pm 7.45$ (before treatment) became $41.78 \pm 8.17$ (after treatment). Overall, the skin tone grade at control and intervention sides was increased from the first time visit until 12 weeks after treatment.

\section{Adverse event}

All participants, experienced no serious side effects (Table 3 ). All patient have mild erythema and subsided in a few days up to 2 weeks without any intervention treatment. Seven participant felt mild pain but can still withhold without any treatment added. The crust peel off in 5-7 days after laser treatment. No post-inflammatory hyperpigmentation found.

Table 2. Results from skin analyzer (Janus) measurement (before and after).

\begin{tabular}{lccc}
\hline Variable & Total $(\mathbf{N})$ & Mean Control \pm SD & Mean Intervention \pm SD \\
Pore & & & \\
$\quad$ Before & 9 & $48.3333 \pm 4.21307$ & $50.0000 \pm 5.59017$ \\
$\quad$ After & 9 & $47.1111 \pm 3.68932$ & $47.2222 \pm 3.45607$ \\
Wrinkle & & & \\
$\quad$ Before & 9 & $19.7778 \pm 3.89801$ & $25.1111 \pm 14.83614$ \\
After & 9 & $18.6667 \pm 4.63681$ & $25.5556 \pm 25.16004$ \\
\hline Spot (UV) & & & \\
$\quad$ Before & 9 & $13.1111 \pm 6.99007$ & $14.2222 \pm 8.05881$ \\
$\quad$ After & 9 & $11.4444 \pm 4.06544$ & $10.4444 \pm 3.16667$ \\
Skin Tone & & \\
$\quad$ Before & 9 & $44.1111 \pm 2.71314$ & $44.7778 \pm 7.44610$ \\
After & 9 & $46.2222 \pm 1.78730$ & $41.7778 \pm 8.16667$ \\
\hline
\end{tabular}

Table 3. Adverse events in patient with fractional erbium laser plus topical AMSC versus Physiologic solution.

\begin{tabular}{lcc} 
Adverse events $\mathbf{N}=9$ & Normal Saline $(\mathrm{n})(\%)$ & AMSC-NP (n) (\%) \\
Mild pain & $7(77.77)$ & $7(77.77)$ \\
Mild erythema & $9(100)$ & $9(100)$ \\
\hline Acne eruption & $2(22.22)$ & $2(22.22)$ \\
\hline
\end{tabular}




\section{Discussion}

Facial rejuvenation is always a hot topic in the field of cosmetic dermatology. A variety of mechanisms causing skin aging have been proven, including declining growth capacity of skin tissue, decreased cell viability, disordered keratinocyte, reduced synthesis of collagen fiber in the dermis layer, and degeneration of elastic fibers. ${ }^{1}$ The desire to maintain or restore a youthful appearance has become an obsession for everyone who concern about it.

A number of treatment have been developed to fight and slow age-related diseases. ${ }^{1}$ Various combination of regenerative dermatologic treatment need to be tested to increase the treatment effect and lessen adverse reactions. ${ }^{8}$ Our study is to prove whether the combination of fractional erbium:YAG laser with topical AMSC metabolite product is effective and safe for facial aging in Asian skin (Figure 1).

The use of fractioned resurfacing to create micro-channels in the epidermis to improve drug delivery into skin is a new concept of treatment called trans-epidermal drug delivery. ${ }^{4}$

Cell-based therapies using the body's own stem cells and growth factors have recently been used as an alternative therapeutic strategy to repair damaged tissue, including skin rejuvenation. ${ }^{2}$ Stem cells have the ability to renew themselves as well as differentiate into specialized cell types and their role has been demonstrated in tissue regeneration. ${ }^{1,7}$ Furthermore, stem cells synthesize and secrete a variety of extracellular matrix proteins, cytokines, growth factors, and other bioactive proteins that contribute to the healing process. A previous study revealed that human embryonic stem cells (hESC-EPC) accelerates wound healing and increases the tensile strength of wounds after topical treatment and subcutaneous injection. In vitro, hESC-EPC conditioned medium (CM) significantly improved the proliferation and migration of dermal fibroblasts and epidermal keratinocytes, and also increased collagen synthesis by fibroblast. ${ }^{2}$ In addition, conditioned media from adipose-derived stem cells (ADSC CM) have shown to inhibit melanogenesis by downregulating tyrosinase and tyrosinase-related protein-1 expression in B16 melanoma cells, demonstrating the whitening effects of ADSCs. Similarly, hESC-EPC CM inhibited melanogenesis in B16 melanoma cells (unpublished data). ${ }^{2}$ Our study referenced a split-face experiment to avoid individual differences, and to ensure that skin had basically the same status before treatment.
Besides using the Glogau scale, we also use skin analyser, Janus, to analyze the grade of skin photo-aging and to make an evaluation after treatment (Figure 2). The data of subjective feelings and objective judgements both demonstrated that the experimental group had a better improvement of pores, and spot UV (pigmentation). Wrinkle have a very slight improvement in intervention side maybe because Janus do not differentiate between the superficial and deep wrinkles, and also wrinkle happened deeper than pore and skin pigmentation in the skin, so it is need more power full laser fractional setting to create deeper micro-channels. Micro-channels can be produced by fractionated laser systems within the skin that can be manipulated in terms of depth and collateral thermal injury. The laser parameters such as energy, pulse width, number of pulses, spot size, and wavelength all play a critical role in determining the resultant effect on the target. Additionally, skin appendages including glands, hair, and vasculature may alter the efficacy of the laser. ${ }^{5}$ Both the Er:YAG and CO2 lasers histologically have been shown to generate coagulated colums in the skin, with a single pass treatment. ${ }^{5}$
The improvement of skin tone also gave the same result between the experimental and control side, maybe because of the multifactorial in skin tone. Skin tone shows a grade of darkness on overall skin surface. Skin tone become not uniform maybe because it is influenced by multifactorial causes, such as stress, pigmentation, sun burn, keratin, and other skin problems. ${ }^{9}$

The benefit of utilizing laser technology is that one can manipulate the depths at which the laser micro-channels are generated. It should be noted is that the more superficial the laser depth, the shorter the recovery time in terms of re-epithelialisation. Conversely, the deeper the micro-channel, the greater the thermal injury and therefore, the longer the down time. ${ }^{5}$ In this study, we used the low power setting of fractional erbium laser for skin rejuvenation and also only one pass each time of treatment, is to shorter the recovery time and also to reduce the adverse reaction such as long term erythema and post inflammatory hyperpigmentation (PIH).

In this study, we used fractional erbium laser because of fewer side effects such as post-inflammatory hyperpigmentation, prolong erythema and less pain but still can get
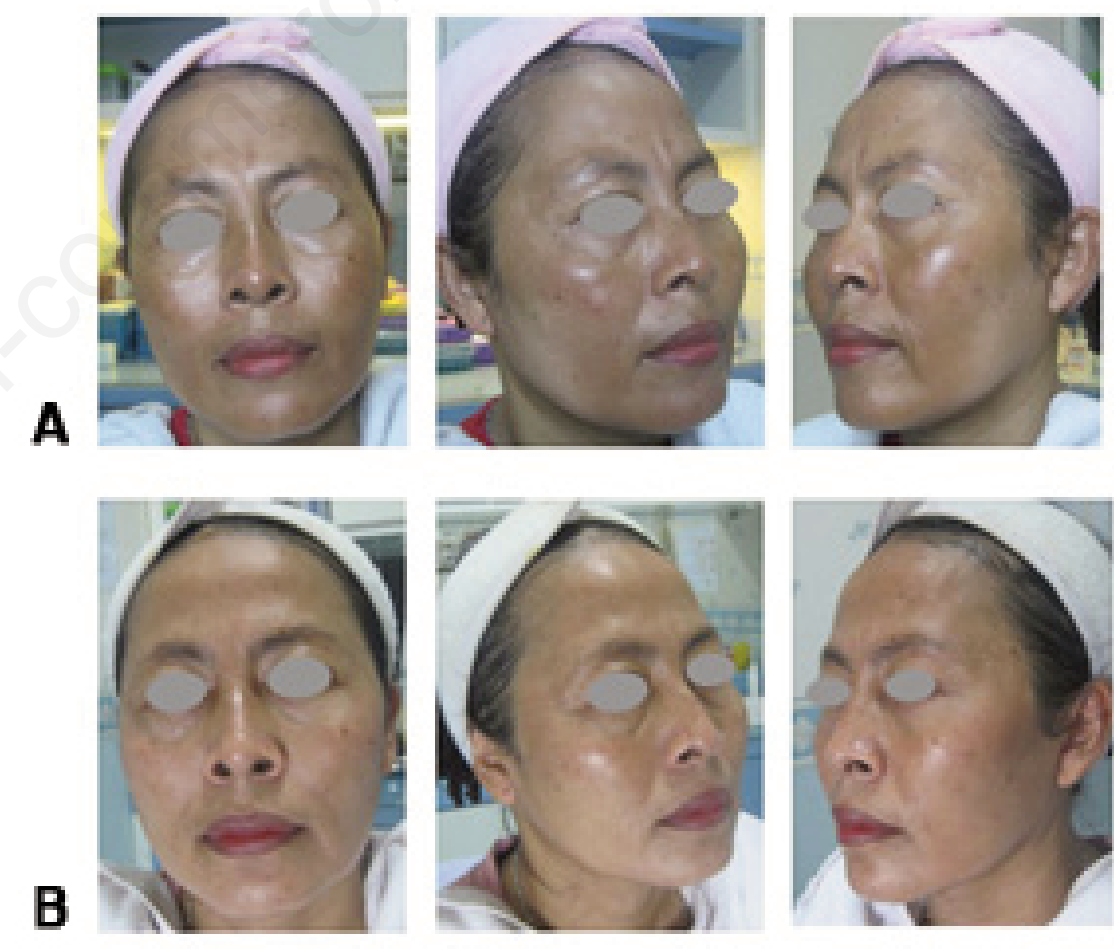

AMSC side (R)

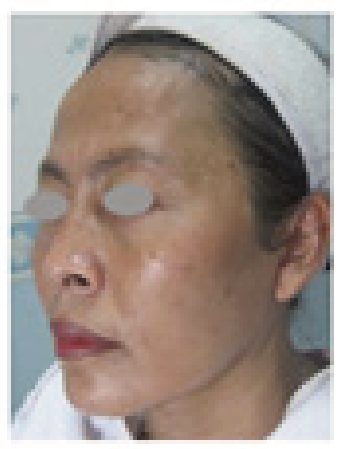
Normal Saline side (L)

Figure 1. Photograph results (A) before the fractional erbium laser procedures and AMSC-MP topical at 0 week and (B) after the same procedures at 4 weeks after the last procedure (16 weeks). 
A
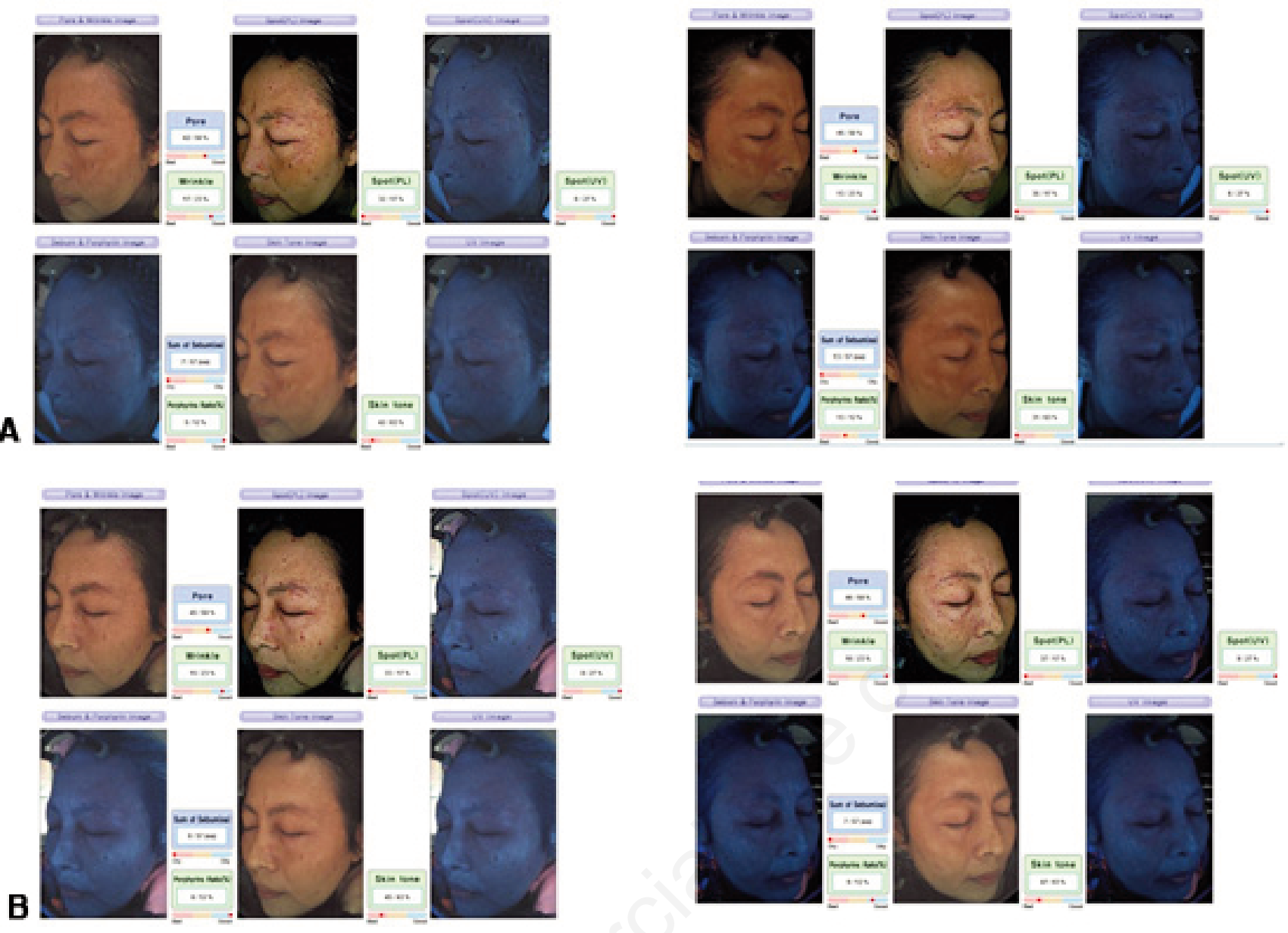

Figure 2. The four indicators of aging skin like pores, wrinkles, spot UV and skin tone were evaluated using skin analyzer, Janus, showed that pores and spot UV give a good improvement in AMSC-MP sides. The wrinkles and skin tone give the same results on both sides. (A) Before the procedures of fractional laser and AMSC-MP topical, right-intervention side (AMSC-MP topical) and left-control side (normal saline). (B) After the procedure of fractional laser and AMSC-MP topical, right-intervention side \& left-control side.

the therapeutic effect, so it is safe for Asian skin. All 9 participants completed the 24week study, experience no serious adverse events. Mild pain and temporary erythema during and after treatments were tolerable in all participants. Two participant had a mild acne eruption but resolved in several days after they applied the tretinoin $0.1 \%$ again and clindamycin $2 \%$ gel.

\section{Conclusions}

The result of this study suggest that fractional erbium YAG laser treatment combined with topical AMSC -metabolite product is safe and effective and maybe a good treatment option for facial rejuvenation in Asian patients.

This study demonstrated that fractionated erbium laser through its discrete injury to the stratum corneum generates micro-channels through which amniotic membrane stem cells could be delivered trans dermally and can enhanced the effect of topically applied AMSC absorbed, thus proving that these micro-channels can be used for transdermal drug delivery to improve the skin aging problem.

It is also need an eligible sample and RCT with the same baseline for the further investigation on the efficacy of fractionated laser plus topical AMSC -metabolite product for facial rejuvenation.

\section{References}

1. Hui Q, Chang P, Guo B, Zhang Y, Tao K. The Clinical Efficacy of Autologous Platelet-Rich Plasma Combined with Ultra-Pulsed Fractional CO2 Laser Therapy for Facial Rejuvenation. Rejuvenation Res. 2017 Feb 1;20(1):25-31 PMCID: PMC5314998 .

2. Lee HJ, Lee EG, Kang S, Sug JH, Chung HM, Kim DH. Efficacy of microneedling plus human stem cell conditioned medium for skin rejuvenation: a randomized, controlled, blinded split-face study. Ann Dermatol 2014; 26: 584-92.

3. Di Germanio C, Bernier M, de Cabo R, Barboni B. Amniotic Epithelial Cells: A new Tool to Combat Aging and AgeRelated Diseases? Front. Cell Dev. Biol. 2016 Nov 4:135

4. Issa MCA, Pires M, Silveira P, de Brito EX, Sasajima C. Transepidermal Drug Delivery: A New Treatment Option for Areata Alopecia. J of Cosm and Laser Therapy. 2015; 17:37-40.

5. Oni G, Lequeux C, Cho M, Zhang D, Lazcarno E, Brown S, Kenkel J. The use of a fractional ablative laser to deliver adipocyte derived stem cells transdermally - a feasibility pilot study. Aesthet Surg J. 2013 Jan;33(1):109-16 PMID:23277622.

6. Kim JE, Kim JK, Ko JY, Ro YS, Chang 
SE. Topical Application of Whitening Agents after Erbium-doped Yttrium Aluminum Garnet Fractional Laser Treatment for Melasma in Asians: A Randomized Controlled Split-face Study. Medical Lasers; Engineering, Basic Research, and Clinical Application. 2012 Dec 1(1): 3-10.

7. Xu X, Wang H, Zhang Y, Liu Y, Li Y, Tao K et al. Adipose-derived Stem Cells
Cooperate with Fractional Carbon Dioxide Laser in Antagonizing Photoaging: A Potential Role of Wnt and $\beta$-catenin Signaling. Cell \& Bioscience 2014 May, 4:24 http://www.cellandbioscience.com/content $/ 4 / 1 / 24$

8. Shin MK, Lee JH, Lee SJ, Kim N. Platelet-Rich Plasma Combined with Fractional Laser Therapy for Skin
Rejuvenation. Dermatol Surg 2012;38: 623-630 .

9. Green AC, Hughes MCB, McBride P, Fourtanier A. Factors associated with Premature Skin Aging (photoaging) Before the Age of 55: A Populationbased Study. Dermatology 2011: 222:74-80. 